\title{
Técnicas y usos en la clasificación automática de imágenes
}

\author{
Isidoro Gil Leiva ${ }^{1}$, Pedro Díaz Ortuño ${ }^{1}$ y José Vicente Rodríguez Muñoz ${ }^{1}$ \\ ${ }^{1}$ Facultad de Comunicación, Universidad de Murcia, España, isgil@um.es, diazor@um.es, \\ jovi@um.es
}

Tipo de contribución: comunicación

Resumen: La producción y generación de información visual mediante teléfonos móviles y cámaras es ingente. También y principalmente a través de la teledetección, mediante la obtención de imágenes de la superficie terrestre por medio de aviones, naves espaciales y satélites que captan y sirven datos sobre meteorología, oceanografía, geología, geografía, geolocalización, seguridad, etc. Estos instrumentos de captura de imágenes generan cada día información visual imposible de procesar manualmente, de ahí que se recurra a diversas técnicas y métodos para la extracción automática de conocimientos útiles. Esta revisión bibliográfica, pretende conocer las técnicas y usos de la clasificación automática de imágenes. Para ello, se emplearon las Bases de datos Scopus y WoS para localizar documentos sobre clasificación automática de imágenes publicados entre 2008 y 2018. De los registros resultantes se buscaron los textos completos de los mismos, llevando a cabo un análisis del contenido para averiguar las técnicas más recurrentes y sus aplicaciones. Con todo ello, se hace patente que las tres técnicas más usadas para la clasificación automática de imágenes son los árboles de decisiones, redes neuronales y máquinas de vectores de soporte, siendo la aplicación de la clasificación automática muy variada, con la que se buscan automatizar procesos repetitivos, la inspección y vigilancias complejas, el control y desarrollo urbanístico o el reconocimiento y valoración tras catástrofes naturales, entre otros asuntos.

Palabras clave: Clasificación automática de imágenes; técnicas y aplicaciones; revisión bibliográfica.

\section{Introducción}

Desde las décadas de 1970-1980 los ingenieros, principalmente, han trabajado intensamente en el desarrollo de técnicas y algoritmos para la categorización e identificación de eventos mediante los datos obtenidos. Inicialmente, fue con el tratamiento de textos con el fin de clasificar automáticamente documentos de acuerdo a los términos de los documentos, para pasar posteriormente al tratamiento de sonido e imágenes en sus diferentes formatos.

En la actualidad, la producción de información no textual visual es enorme. Teléfonos móviles y cámaras (ocio, televisión, drones, etc.); la teledetección que toma imágenes de la superficie terrestre mediante aviones, naves espaciales o satélites (hoy en día más de cinco mil satélites sirven datos sobre meteorología, oceanografía, geología, geografía, geolocalización, seguridad, etc.). Estos numerosos instrumentos de captura de imágenes generan cada día información no textual inconmensurable e imposible de procesar manualmente, de ahí que se recurra a técnicas de selección y minería de datos para la extracción automática de conocimientos útiles.

En el caso de las imágenes, se trató de dar soluciones por medio de investigaciones en clasificación automática y, por otro lado, en la recuperación basada en el contenido, CBIR en sus siglas en inglés. 
Así, la disciplina denominada visión por ordenador estudia cómo procesar, analizar e interpretar imágenes de manera automática. La visión por ordenador se apoya en disciplinas y ciencias como la matemática (estadística o geometría), física (óptica), neurobiología (inteligencia artificial) o el procesamiento de señales digitales.

Las herramientas de extracción de conocimiento tratan de extraer e identificar patrones en los datos para hacerlos comprensibles y útiles, la preparación de estos, la identificación de patrones, la interpretación, la visualización y, por último, la obtención de conocimiento. En las herramientas de extracción de conocimiento, la clasificación automática por medio de clasificadores de diferentes tipos desempeña una función nuclear, y viene empleándose en diferentes ámbitos de la vida real como en el ámbito de la economía (datos para la toma de decisiones), biometría (reconocimiento de personas), inspección automática de productos y calidad (separación y empaquetado de frutas, carnes, paquetes, etc.), o en el procesamiento de las mencionadas imágenes procedentes de la teledetección.

La información textual y audiovisual ha venido siendo indizada y clasificada manualmente hasta que su volumen era relativamente manejable. En la década de 2000 se comenzó a trabajar en la anotación automática de imágenes para la asignación de los conceptos que aparecen en las imágenes tras el procesamiento del color, la textura, las formas, los objetos o las acciones. De esta manera se detecta y anota automáticamente palabras como: día, noche, interior, exterior, hombre, bicicleta, río, edificio, vegetación, etc. a partir de una colección de imágenes previamente anotadas que sirven de modelo de aprendizaje al sistema. Se trata, por tanto, de una notación física y no semántica de la imagen.

De igual modo, se viene trabajando en la recuperación de imágenes basadas en contenido (CBIR, en sus siglas en inglés) donde también se aplican clasificadores. Velazco Paredes $(2014$, p. 6) señala los tipos de consulta que se pueden realizar en los sistemas CBIR: consultas por palabras clave, por controles visuales (color, textura, contrastes o brillo), mediante imágenes por comparación o mediante trazos que realiza el usuario que pueden combinarse con color o textura. Por otro lado, los procesos seguidos en el análisis de las imágenes podemos dividirlos en tres niveles:

- Procesos de bajo nivel: adquisición de las imágenes y preparación de las imágenes (mejora y realce de contraste o de bordes), identificación del espacio de color a través de modelos que componen cada color mediante una combinación lineal de los tres colores primarios rojo, verde y azul (RGB) y el modelo HSV (Tono, Saturación y Valor).

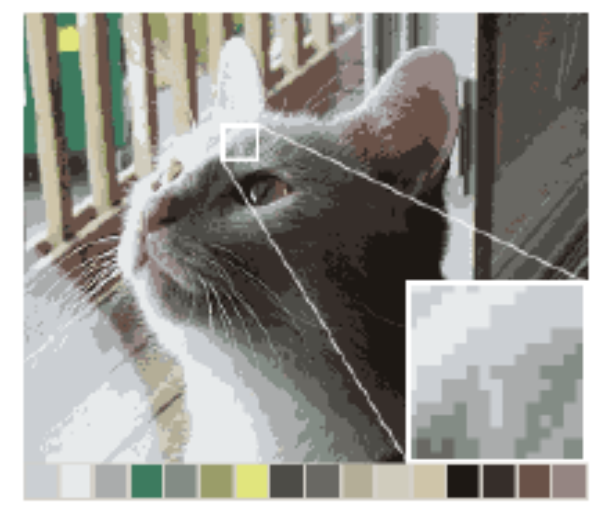

Figura 1: Ejemplo de cuantificación del color. Fuente: Velazco Paredes (2014, p. 16). 
- Procesos de nivel medio: segmentación de la imagen por niveles de grises, bordes, o formas, entre otras y, por otro lado, marcado de las partes de la imagen.

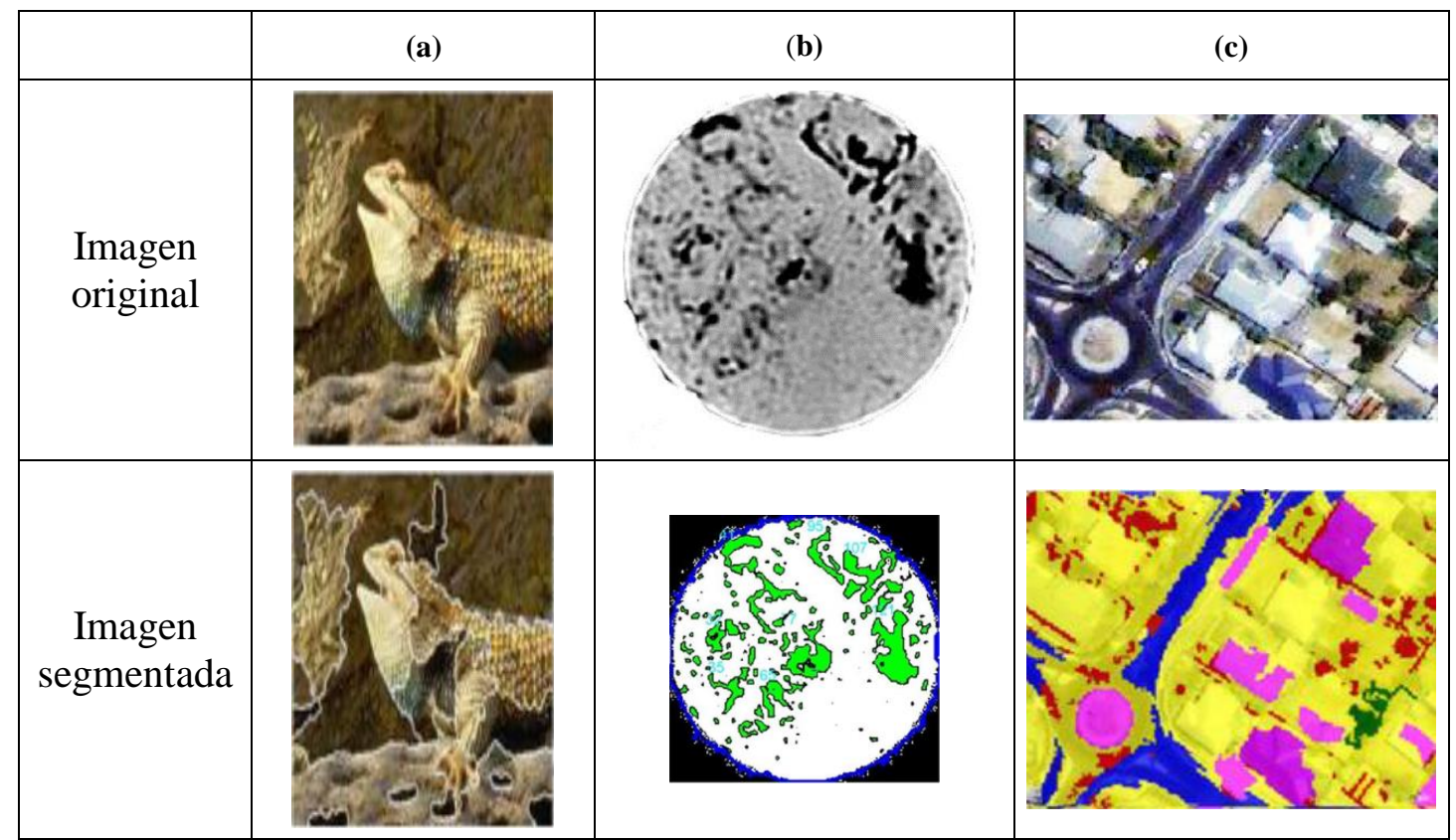

Tabla 1: Ejemplos de segmentación de imágenes. Fuente: (a) Dong ping Tian (2013, p. 390); (b) Binhui et al. (2017, p. 91); (c) Jabari \& Zhang (2013, p. 776).

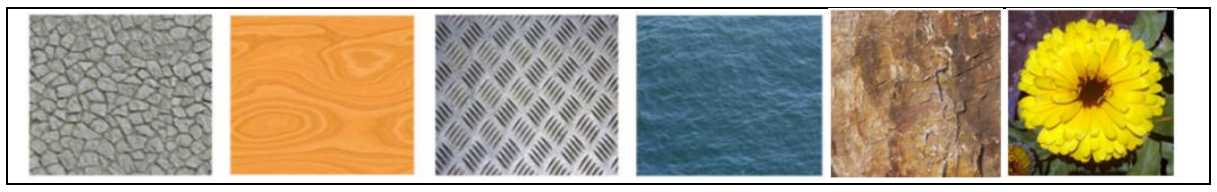

Tabla 2: Ejemplos de texturas en una imagen. Piedra, madera, metal, agua, roca y flor.

- Proceso de nivel alto: reconocimiento de patrones e interpretación usando para ello clasificadores basados en redes neuronales, máquinas de soporte vectorial (SVM, en sus siglas en inglés) o árbol de decisión, entre otros.

En la bibliografía, encontramos diferentes técnicas para la clasificación automática de imágenes:

\begin{tabular}{|l|l|}
\hline S. B. Kotsiantis (2007, 251) & 1. Métodos de aprendizaje basado en la lógica: \\
& Árboles de decisión \\
& Clasificadores basados en reglas \\
& 2. Técnicas basadas en perceptrón \\
& Perceptones de una sola capa \\
& Perceptones multicapa \\
& Redes de función de base radial (RBF) \\
& 3. Métodos de aprendizaje estadístico \\
& Clasificadores Naive Bayes \\
& Redes bayesianas \\
& 4. Aprendizaje basado en instancias \\
& 5. Máquinas de soporte vectorial (SVM) \\
\hline Das, A.J. Saikia, N. y Sarma, K.K. (2016, & Métodos de clasificación automática supervisada: \\
\hline
\end{tabular}




\begin{tabular}{|l|l|}
\hline 268). & Redes neuronales \\
& Boosting Adaptative \\
& Máquinas de vectores de soporte \\
& Árboles de decisión \\
& Lógica borrosa \\
\hline
\end{tabular}

Tabla 3: Técnicas para la clasificación automática de imágenes

\section{Objetivos}

El objetivo de esta revisión bibliográfica es triple, por un lado, revisar las técnicas y métodos de clasificación usados en la última década en la clasificación automática de información no textual; describir someramente las técnicas más empleadas; así como averiguar a qué ámbitos se están dirigiendo dichas investigaciones.

\section{Material y método}

Para lograr los objetivos marcados, en primer lugar se seleccionaron las Base de datos Scopus y WoS para localizar documentos sobre clasificación automática de imágenes publicados entre 2008 y 2018. En la Tabla 4 se detallan las búsquedas empleadas y el número de documentos recuperados.

\begin{tabular}{|c|c|c|}
\hline Base de datos & Ecuación de búsqueda & Publicados 2008-2018 \\
\hline Scopus & $\begin{array}{l}\text { Keywords: "automatic indexing" AND } \\
\text { Keywords: "image classification" = } 44 \\
\text { documentos }\end{array}$ & 32 \\
\hline Scopus & $\begin{array}{l}\text { Keywords: "automatic indexing" AND } \\
\text { Keywords: "image analysis" = } 102 \\
\text { documentos }\end{array}$ & 9 \\
\hline WoS & $\begin{array}{l}\text { Tema: "automatic indexing" AND } \\
\text { Tema: "image analysis" = } 12\end{array}$ & 2 \\
\hline Wos & $\begin{array}{l}\text { Tema: "automatic indexing" AND } \\
\text { Tema: "image classification" = } 11\end{array}$ & 3 \\
\hline & Total & 45 \\
\hline
\end{tabular}

Tabla 4: Búsquedas y resultados obtenidos

De los cuarenta y cinco registros recuperados que cumplían los requisitos de las búsquedas planteadas (temática y período de publicación entre 2008 y 2018 ambos años incluidos), sólo se localizó el texto completo de treinta trabajos. Por tanto, estas treinta publicaciones fueron las manejadas para ejecutar la revisión. Para la recopilación de los datos, durante la lectura, se usó una plantilla donde se consignaron los datos de interés, en este caso, información relativa a las técnicas y métodos de clasificación, el objeto de análisis o el tipo de imágenes manejadas por los autores, entre otros aspectos.

\section{Resultados}

En el análisis de los resultados obtenidos, dentro de los aspectos a analizar, encontramos las siguientes características 
4.1 Técnicas más usadas en la clasificación automática de imágenes. El análisis de los treinta tres artículos publicados entre 2008 y 2018 nos ha permitido averiguar que la mayor parte de los investigadores recurren a técnicas de clasificación supervisada que cuenta con procesos de entrenamiento y aprendizaje antes de la clasificación real de las imágenes; pero también se observa interés en las no supervisadas que no usan conjuntos de datos de entrenamiento. Las técnicas supervisadas más empleadas han sido el árbol de decisión, las redes neuronales y las máquinas de vectores de soporte.

\begin{tabular}{|c|c|}
\hline Autor/es & Clasificación de características visuales semánticas de alto nivel \\
\hline Al-Batah, Isa, Zamli, Sani, y Azizli, 2009 & $\begin{array}{l}\text { Redes neuronales. Red neuronal de perceptrón multicapa en } \\
\text { cascada (c-MLP) }\end{array}$ \\
\hline Amutha y Kavitha, 2011 & $\begin{array}{l}\text { Máquina de vectores de soporte de características ponderadas } \\
\text { (WFSVM) mediante Algoritmo C-Medios Posibilístico basado en } \\
\text { el kernel (KPCM) }\end{array}$ \\
\hline Augereau, Journet, Vialard, y Domenger, 2014 & Máquinas de vectores de soporte \\
\hline Cintra, Novack, Rego, Costa, y Feitosa, 2010 & Árboles de decisión. Algoritmo C4.5 en plataforma Weka 3.7 \\
\hline Correia, Duarte, Teodoro, y Monteiro, 2018 & Clasificación K-Medias no supervisada \\
\hline Dimitrios et al., 2010 & $\begin{array}{l}\text { Nuevo clasificador automático de cuerpos deformados por } \\
\text { comparación de los contornos }\end{array}$ \\
\hline Ghaffarian, 2014 & Clasificación supervisada por paralelepípedos mejorada \\
\hline HaCohen-Kerner et al., 2015 & $\begin{array}{l}\text { Clasificación supervisada: algoritmo J48, Random Forests (RF) y } \\
\text { Sequential Minimal Optimization (SMO). }\end{array}$ \\
\hline Hao, Ge y Wang, 2018 & Máquinas de vectores de soporte \\
\hline Hemsley, Mukundan, 2009 & Clasificación usando Multifractal Spectra. Técnicas de clustering. \\
\hline Hermosilla, Ruiz, Recio, y Estornell, 2011 & Árboles de decisión (Clasificador C5.0) \\
\hline Jabari y Zhang, 2013 & $\begin{array}{l}\text { Reglas difusas, sistema de inferencia de eCognition: Fuzzy Image } \\
\text { Classification }\end{array}$ \\
\hline Jain, 2013 & Máquina de vectores de soporte. Redes neuronales \\
\hline $\begin{array}{l}\text { Kupidura, Osińska-Skotak, y Pluto- } \\
\text { Kossakowska, } 2016\end{array}$ & Modelo "GROUPS" \\
\hline Li et al., 2017 & Máquina de vectores de soporte \\
\hline Lin, $\mathrm{Li}, \mathrm{Yu}, \mathrm{y}$ Wu, 2018 & Máquina de aprendizaje extremo ( ELM) (Algoritmo genético) \\
\hline Ma, Zeheng, Eitel y Moskal, 2016 & $\begin{array}{l}\text { Algoritmo basado en geometría (GAFPC), mejora del método de } \\
\text { Lalonde }\end{array}$ \\
\hline Minetto, Thome, Cord, Leite y Stolfi, 2014 & Máquinas de vectores de soporte \\
\hline Morioka et al., 2016 & Clasificador de tabla de decisiones \\
\hline Murphy, Maggioni, 2018 & Aprendizaje de difusión espectral-espacial (DLSS) \\
\hline Raj y SivaSathya, 2016 & $\begin{array}{l}\text { Redes neuronales. Algoritmos inspirados en la Biología, red } \\
\text { neuronal de perceptrón multicapa (MLPNN), mapas auto- } \\
\text { organizados (SOM), C-Medias borroso. }\end{array}$ \\
\hline Rezaeian, 2012 & $\begin{array}{l}\text { Máquina de vectores de soporte. Clasificador k vecinos más } \\
\text { cercanos (k-NN); Clasificador de Bayes ingenuo }\end{array}$ \\
\hline $\begin{array}{l}\text { Senthilnath, Kulkarni, Benediktsson, y Yang, } \\
2016\end{array}$ & $\begin{array}{l}\text { Agrupamiento K-Medias; Algoritmo Genético (GA); (Software } \\
\text { Matlab) }\end{array}$ \\
\hline Shen, 2009 & Redes neuronales \\
\hline Yang, Huang, Kuo, Tsai, y Lin, 2017 & Árboles de decisión \\
\hline Tian, 2013 & $\begin{array}{l}\text { Revisión de desarrollos en extracción de características de imagen } \\
\text { y sus técnicas de representación }\end{array}$ \\
\hline You, Wang, Ma, Chen, Wang, Shen y Liu, 2018 & Algoritmo MBI \\
\hline Zhang \& Zhang, 2011 & Redes neuronales. Lógica difusa \\
\hline Zhang, Hu, Jing, Woods, y Courville, 2008 & Árboles de decisión \\
\hline
\end{tabular}

Tabla 5: Técnicas para la clasificación de alto nivel 
4.2 Descripción de las técnicas más usadas en la clasificación automática de imágenes. A continuación, se describen sucintamente las tres técnicas de clasificación supervisadas más usadas para la clasificación automática de las características visuales semánticas de las imágenes.

4.2.1 Árboles de decisión. Esta técnica surge a mitad de la década de 1940 de la teoría de juegos de John Von Neumann y Oskar Morgenstern. Posteriormente, con la Informática se incorporan los conjuntos de entrenamiento. En el ámbito de la inteligencia artificial, son usados los árboles de decisión para enseñar a un algoritmo a comportarse como un humano ante una situación determinada. Un árbol de decisión es una representación en forma jerárquica de una decisión y de todas sus consecuencias. Ayudan a tomar la decisión más acertada ante varias posibles y así alcanzar la resolución de un problema según la mejor probabilidad. Este proceso decisorio se visualiza en un modelo gráfico arbóreo con un sistema de notación compuesto por cuadrados (nodo de decisión que representa el momento puntual de la decisión y se extiende en tantas ramas como alternativas posibles existan); círculos (nodo de incertidumbre y probabilidad que muestra los posibles resultados); y flechas (ramificación de las alternativas donde cada línea indica la probabilidad de un posible resultado. Las probabilidades de dos alternativas suman siempre 1). Un conjunto de ramas constituyen un camino por donde se van tomando decisiones para la resolución de un problema, que se puede conformar como una regla de clasificación.

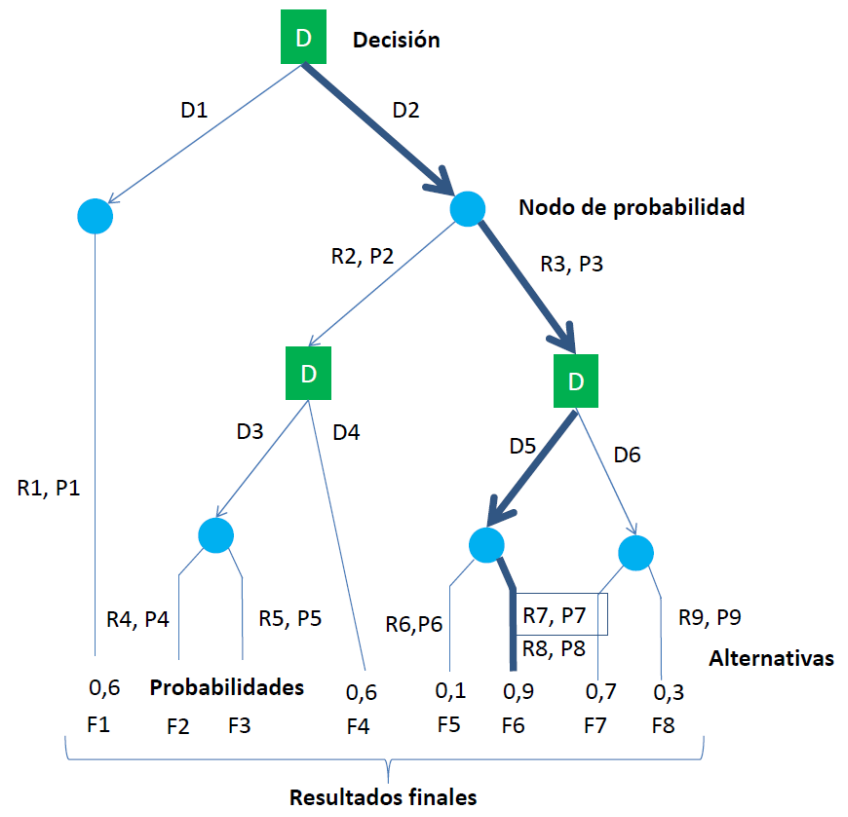

Figura 2: Representación de un árbol de decisión

4.2.2 Redes neuronales. La modelización mediante redes neuronales busca simular capacidades humanas como la de asociación de hechos y, de este modo, aplicar la experiencia acumulada. Se trata de una simulación abstracta de los sistemas nerviosos biológicos. El neurofísico Warren McCulloch y el matemático Walter Pitts en 1943 propusieron el primer modelo computacional basado en la actividad nerviosa mediante circuitos eléctricos. Una clasificación básica de los modelos neuronales los divide en modelos biológicos (tratan de simular sistemas neuronales biológicos) y modelos artificiales (redes artificiales aplicadas). Los modelos de red neuronal de tipo biológico están modelizados como una regla de propagación de señales con una entrada, una toma de decisiones y una salida. 
Los modelos de redes neuronales artificiales tienen la capacidad de aprender a realizar tareas mediante una experiencia inicial o entrenamiento; capacidad para la auto-organización tras la fase de aprendizaje; capacidad de operar en tiempo real debido al uso de un gran número de nodos con un alto grado de interconectividad; y capacidad para realizar un procesado no lineal que permite clasificar patrones y aumentar su inmunidad a cometer fallos ante situaciones distorsionadas o datos incompletos. La estructura de la neurona artificial está compuesta por nodos o unidades. En la Figura 3 se muestran sus componentes.

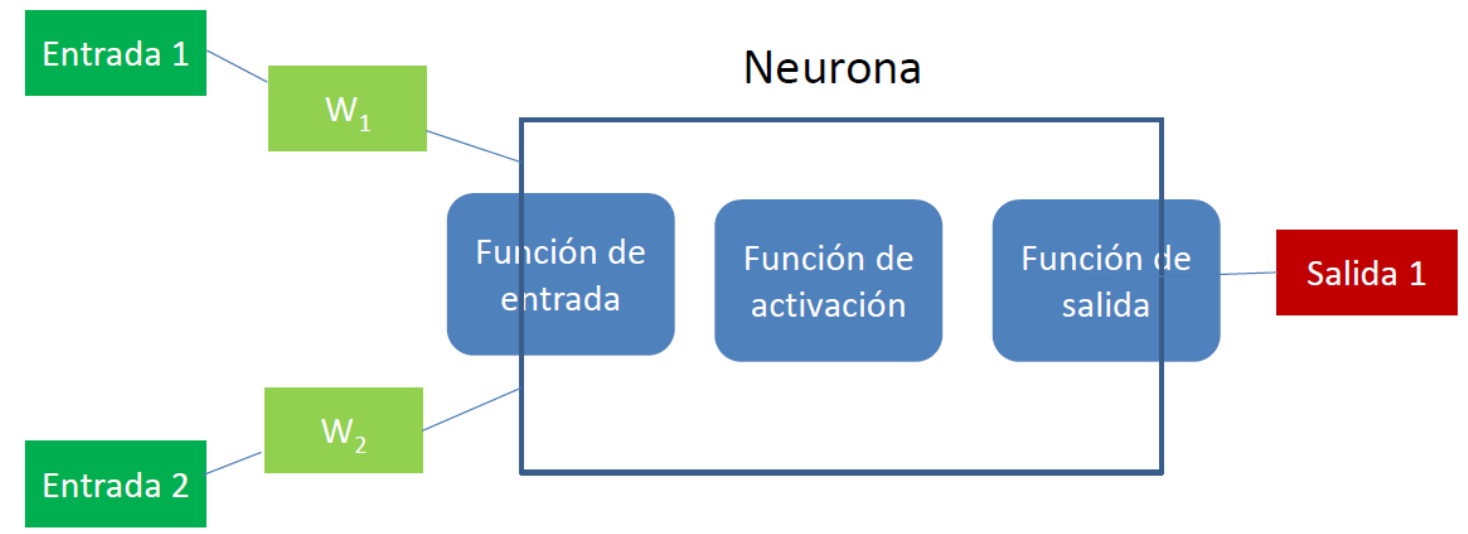

Figura 3: Componentes de una red neuronal artificial

La función de entrada reduce todos los valores de entrada $\left(\mathrm{W}_{1}, \mathrm{~W}_{2}, \mathrm{~W}_{3}\right.$, etc. $)$ a un único valor, que se denomina entrada global, y se obtiene mediante una función (sumatorio, producto o el valor de entrada mayor), multiplicados por sus correspondientes pesos. La función de activación calcula el valor o estado de actividad de una neurona que puede ser $(0,1)$ ó $(-1,1)$ mediante el cómputo de una función, siendo las más usadas la función umbral, lineal, sigmoidea o tangente hiperbólica. El tercer elemento de una red neuronal es la función de salida que proporciona la respuesta o valor de salida que puede ser transmitido a otras neuronas vinculadas. Las redes neuronales se organizan en capas y cada capa contiene un número variable de neuronas. La capa de entrada que recibe directamente la información de fuentes externas a la red; las capas ocultas, que se encuentra entre la de entrada y la salida y no mantienen contacto alguno con el exterior (puede ser de una sola capa o multicapa); y por último, la capa de salida que envía información de la red hacia el exterior.

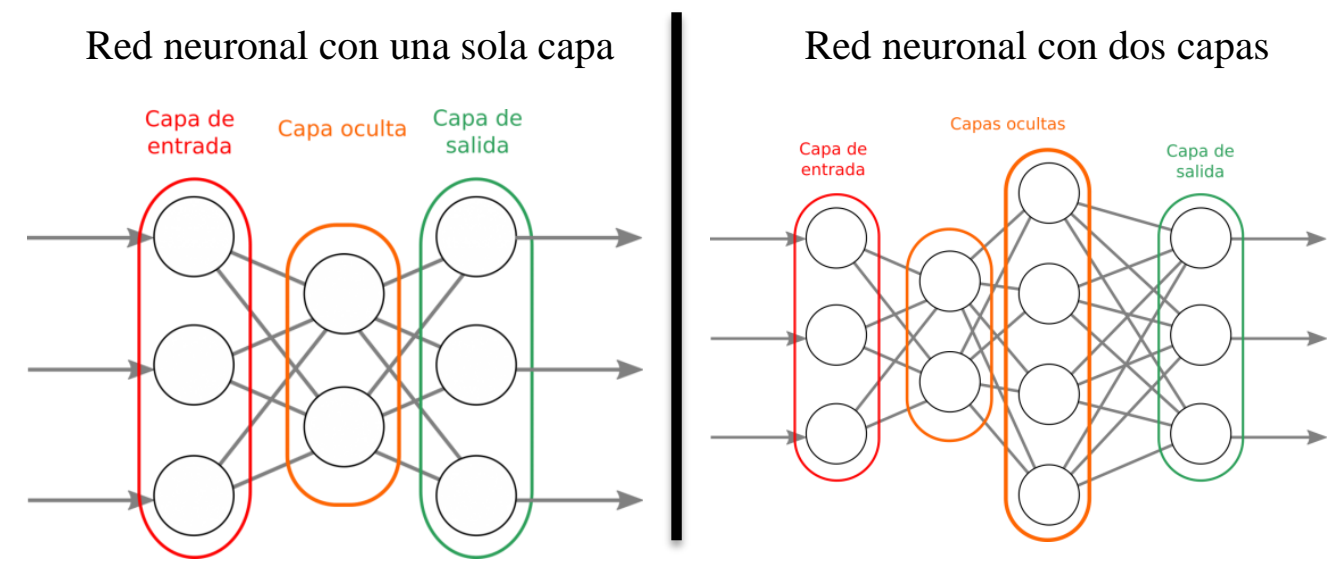

Figura 4: Capas en una red neuronal artificial. Fuente: https://robologs.net/2017/01/22/tutorial-de-redesneuronales-con-vrep-c-y-linux/ 
El conocimiento en una red neuronal está distribuido por todo el sistema y el aprendizaje en una red implica un cambio constante en los pesos de las conexiones entre neuronas, lo que significa la destrucción, modificación y creación de conexiones entre ellas. Los pesos representan la información usada por la red para resolver un problema, y cuando los pesos permanecen estables significa que el proceso ha finalizado y por tanto, la red ha terminado el aprendizaje.

Hay dos fases en la modelización con redes neuronales: fase de entrenamiento y aprendizaje (se emplean al menos dos conjuntos de datos y patrones con ciertas variaciones para definir un modelo a partir de unos datos de entrada y una salida deseada) y fase de prueba (se persigue minimizar el error entre la salida del modelo y lo deseado).

4.2.3 Máquinas de vectores de soporte. Las máquinas de vectores soporte (SVM, en sus siglas en inglés) fue introducida en la década de 1990 por Vladimir Vapnik y sus colegas de laboratorio desde la teoría del aprendizaje estadístico, para resolver problemas de regresión y clasificación binaria lineal o hiperplano usando las funciones kernel. La SVM se ha utilizado en la categorización de textos, el reconocimiento de grafías o la clasificación de imágenes.

La modelización de SVM a partir de unas entradas determinadas busca establecer dos clases separadas por una línea y un hiperplano representadas con puntos en un espacio bidimensional. Una SVM es un algoritmo que a partir del producto escalar de los vectores multidimensionales de las muestras construye un hiperplano o conjunto de hiperplanos y un espacio de dimensionalidad muy alta que separa los grupos. Con el hiperplano se trata de encontrar el hiperplano óptimo de separación entre las clases; es decir, el plano para el cual la separación entre clases es máxima. El hiperplano óptimo se define por una línea recta.

En la Figura 5 se muestran los hiperplanos de separación en un espacio bidimensional de un conjunto de datos separables en dos clases. En la izquierda se ofrecen los posibles planos, en el centro, el hiperplano de separación óptimo, y a la derecha, un ejemplo de clasificación automática de imágenes.

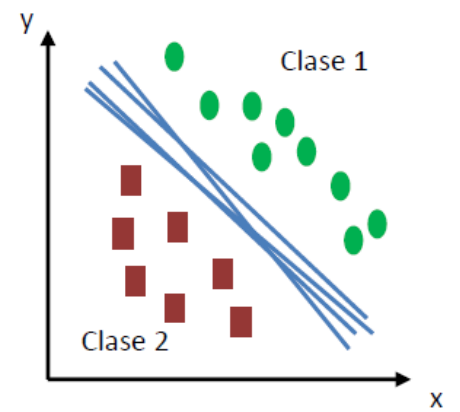

Posibles hiperplanos de separación
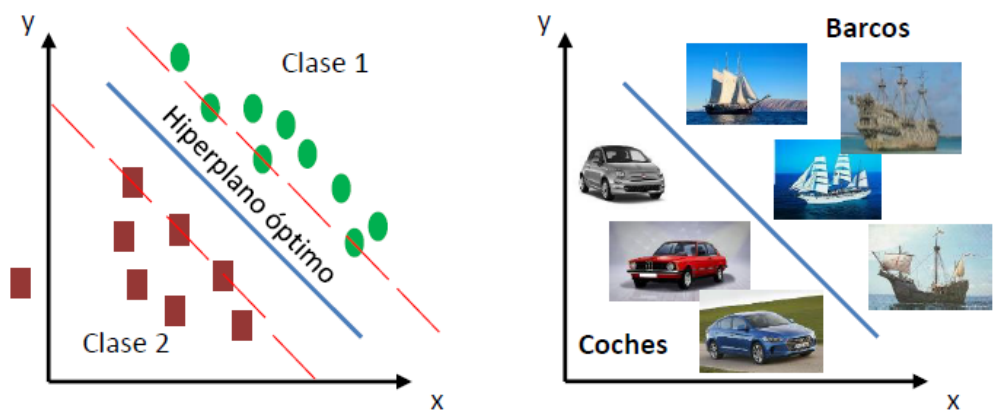

Hiperplano de sepación óptimo

Figura 5: Hiperplanos de separación en un espacio bidimensional

Para la modelización de las SVM se requiere una fase inicial de entrenamiento y aprendizaje. Una vez conformada la modelización el sistema está listo para recibir nuevas entradas y buscar la correspondencia entre el modelo y las entradas para clasificarlas.

Las tres técnicas que acabamos de describir brevemente emplean alguno de estos tres tipos de aprendizaje para la resolución de problemas: a) aprendizaje supervisado: Se suministra un conjunto 
de datos de entrenamiento con ejemplos ya resueltos en forma de pares de datos que representa el comportamiento, el grupo o el valor adecuados, es decir, el problema y la solución. Tras el proceso de aprendizaje se suele emplear un segundo conjunto de pares de datos para validar la tasa de error. El entrenamiento es un proceso iterativo de adaptación de tal manera que el resultado se acerque a las salidas deseadas; b) aprendizaje no supervisado: No se proporcionan las salidas esperadas. La red maneja los datos de entrada y un conjunto de reglas para encontrar patrones de los que debe aprender; y c) aprendizaje con refuerzo: Se aprende a través de un proceso de ensayo y error y un sistema de recompensa/penalización de acuerdo a los resultados que se van logrando.

Para finalizar este apartado se esquematiza la secuenciación de los procesos ejecutados en la clasificación automática de imágenes.

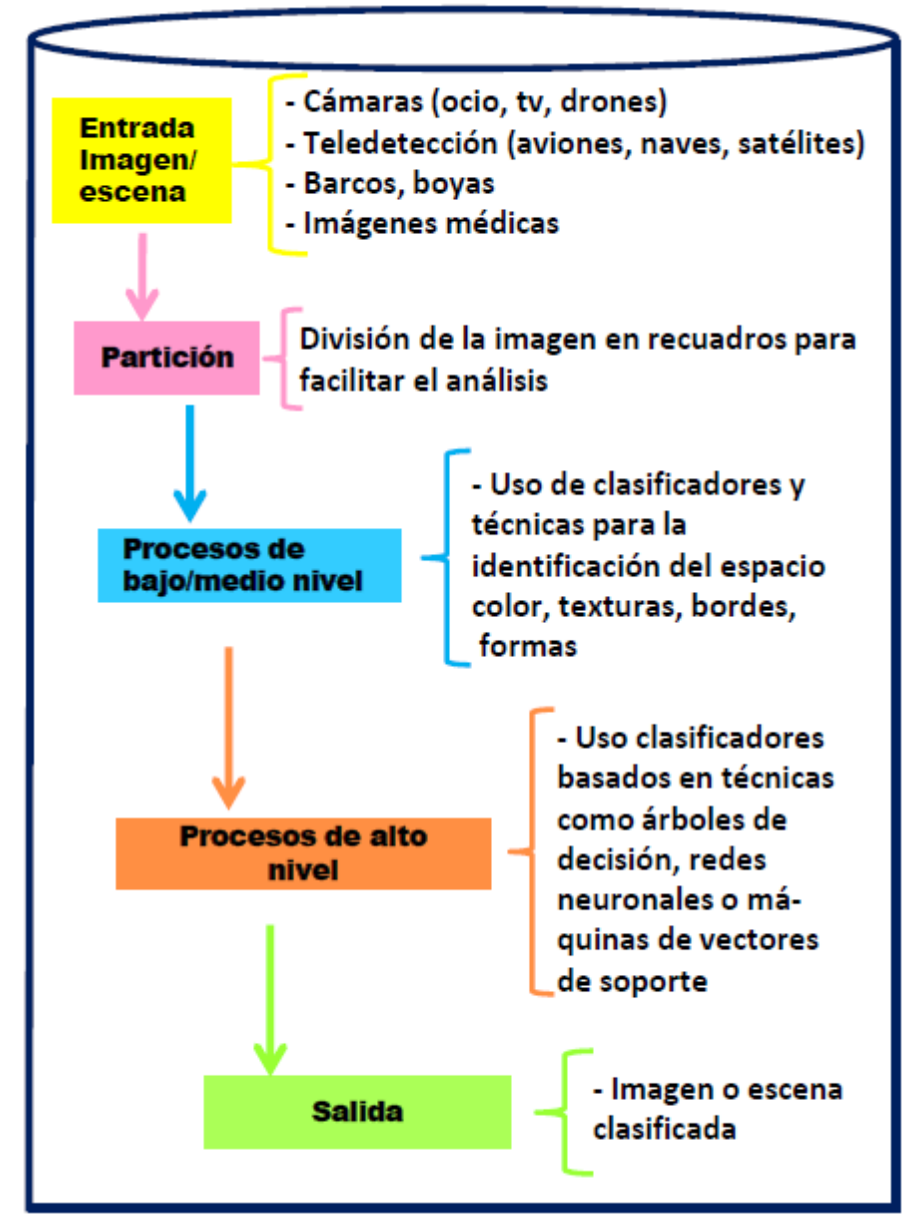

Figura 6: Procesos en la clasificación automática de imágenes

4.3 Objeto de estudio y aplicaciones de la clasificación automática de imágenes. El análisis de los treinta textos que conforma la muestra para la realización de esta revisión también ha permitido identificar los tipos de imágenes con las que se está trabajando, así como el objeto de estudio (Tabla $6)$.

La mayor parte de las imágenes manejadas para las investigaciones provienen de la teledetección (satélites) aunque también se usan imágenes procedentes de microscopios o de cámaras móviles para captar escenas urbanas. La aplicación de la clasificación automática es muy variada y se busca la automatización de procesos repetitivos (como, por ejemplo, la clasificación de hojas del tabaco 
por calidades); inspección y vigilancia complejas (estado y evolución del hielo marino, cobertura del suelo, biomasa arbórea en bosques, identificación de especies forestales, etc.); inspección, control y desarrollo urbanístico; reconocimiento y valoración de zonas después de catástrofes naturales (daños en edificios o pérdidas de cosechas, etc.) entre otros asuntos.

\begin{tabular}{|c|c|}
\hline Autores & Aplicaciones y usos \\
\hline Al-Batah, Isa, Zamli, Sani, y Azizli, 2009 & $\begin{array}{l}\text { Clasificación automática de características (forma, tamaño y textura de } \\
\text { la superficie) de los agregados del hormigón }\end{array}$ \\
\hline Amutha y Kavitha, 2011 & Clasificación automática de imágenes (genérico) \\
\hline $\begin{array}{l}\text { Augereau, Journet, Vialard, y Domenger, } \\
2014\end{array}$ & Clasificación de imágenes en documentos \\
\hline $\begin{array}{l}\text { Cintra, Novack, Rego, Costa, y Feitosa, } \\
2010\end{array}$ & $\begin{array}{l}\text { Clasificación de la cobertura del suelo para el monitoreo de la selva } \\
\text { tropical }\end{array}$ \\
\hline Correia, Duarte, Teodoro, y Monteiro, 2018 & $\begin{array}{l}\text { Procesamiento y clasificación de imágenes de sensores remotos y } \\
\text { satélites }\end{array}$ \\
\hline Dimitrios et al., 2010 & $\begin{array}{l}\text { Identificación y clasificación de parásitos en imágenes microscópicas } \\
\text { 2D }\end{array}$ \\
\hline Ghaffarian, 2014 & Detección de edificios \\
\hline HaCohen-Kerner et al., 2015 & $\begin{array}{l}\text { Clasificación de documentos en categorías en diferentes categorías } \\
\text { (salud, estilo de vida, ocio, naturaleza, medioambiente y política) }\end{array}$ \\
\hline Hao, Ge y Wang, 2018 & Anotación de imágenes mediante conceptos \\
\hline Hermosilla, Ruiz, Recio, y Estornell, 2011 & Detección y localización de edificios \\
\hline Hughes, 2009 & Clasificación de tipos de hielo marino \\
\hline Jabari y Zhang, 2013 & $\begin{array}{l}\text { Clasificación de imágenes urbanas satelitales (sombra, vegetación, } \\
\text { camino, edificación y tierra desnuda) }\end{array}$ \\
\hline Jain, 2013 & Clasificación automática de imágenes basado en el contenido (CBIR) \\
\hline $\begin{array}{l}\text { Kupidura, Osińska-Skotak, y Pluto- } \\
\text { Kossakowska, } 2016\end{array}$ & $\begin{array}{l}\text { Clasificación automática de imágenes satelitales VHR (agua, bosque, } \\
\text { baja vegetación, suelo desnudo y área urbana) }\end{array}$ \\
\hline Li et al., 2017 & Clasificación automática de fracturas y vulgos en rocas \\
\hline Lin, Li, Yu, y Wu, 2018 & $\begin{array}{l}\text { Imágenes de detección remota (tierras desnudas, humedales, agua, } \\
\text { construcción, tierras cultivables, tierras forestales y algas) }\end{array}$ \\
\hline Ma, Zeheng, Eitel y Moskal, 2016 & $\begin{array}{l}\text { Clasificación del dosel de un bosque (biomasa de madera y hoja y } \\
\text { espacio ocupado por un árbol o zona de árboles) }\end{array}$ \\
\hline Minetto, Thome, Cord, Leite y Stolfi, 2014 & $\begin{array}{l}\text { Detección y extracción de texto legible en escenas visuales urbanas } \\
\text { (carteles publicitarios, luminosos publicitarios, toldos, etc.). }\end{array}$ \\
\hline Morioka et al., 2016 & Identificación de riesgo de aneurismas aórticos abdominales \\
\hline Hemsley, Mukundan, 2009 & $\begin{array}{l}\text { Clasificación de imágenes microscópicas de tejidos pulmonar, hepático } \\
\text { y arterial }\end{array}$ \\
\hline Murphy, Maggioni, 2018 & Segmentación de imágenes hiperespectrales. \\
\hline Raj y SivaSathya, 2016 & $\begin{array}{l}\text { Clasificación mediante diferentes formas espectrales, índices de } \\
\text { vegetación, información contextual, imágenes multitemporales o datos } \\
\text { multisensor }\end{array}$ \\
\hline Rezaeian, 2012 & Detección y clasificación de edificios dañados por terremotos \\
\hline $\begin{array}{l}\text { Senthilnath, Kulkarni, Benediktsson, y } \\
\text { Yang, } 2016\end{array}$ & $\begin{array}{l}\text { Clasificación de tipos de cultivo: áreas problemáticas y tamaño de zona } \\
\text { afectada; cambios estacionales y anormalidades en la vegetación; } \\
\text { estimación temprana del rendimiento del cultivo. }\end{array}$ \\
\hline Shen, 2009 & $\begin{array}{l}\text { Identificación automática del autor de una pintura de los siglos XVI al } \\
\text { XVIII }\end{array}$ \\
\hline Yang, Huang, Kuo, Tsai, y Lin, 2017 & $\begin{array}{l}\text { Estimación de la cantidad de arroz en cultivos de arrozales para } \\
\text { tasaciones tras catástrofes }\end{array}$ \\
\hline $\begin{array}{l}\text { You, Wang, Ma, Chen, Wang, Shen y Liu, } \\
2018\end{array}$ & Detección y delimitación de edificios en zonas urbanas \\
\hline Zhang \& Zhang, 2011 & Clasificación de hojas de tabaco para agilizar los procesos de selección \\
\hline Zhang, Hu, Jing, Woods, y Courville, 2008 & Clasificación de especies forestales \\
\hline
\end{tabular}

Tabla 6: Usos y aplicaciones de la clasificación automática 


\section{Conclusiones}

En primer lugar, hay que señalar que la producción de información no textual es en la actualidad ingente y que este fenómeno no va a dejar de crecer de modo exponencial en adelante. Las cámaras fijas y móviles en todas sus manifestaciones, así como la teledetección con la toma imágenes de la superficie terrestre mediante aviones o dispositivos espaciales (pensemos que hoy, más de cinco mil satélites sirven datos sobre meteorología, oceanografía, geología, geografía, geolocalización, seguridad, etc.), son instrumentos que suministran petabytes de datos cada día, imposible de procesar manualmente. Debido a esta enorme cantidad de imágenes se recurre a técnicas para la extracción automática de información útil y procesable para la toma de decisiones y el consiguiente aumento del conocimiento de los fenómenos con los que tratamos diariamente.

La mayor parte de las imágenes manejadas para las investigaciones provienen de la teledetección (satélites), pero la variedad de otros dispositivos es patente, entiéndase, imágenes procedentes de microscopios, para el campo de la Ciencias naturales, así como el de las cámaras fijas o móviles para captar escenas urbanas, tanto humanas como de territorio.

Hay que destacar, que las tres técnicas más usadas en la clasificación automática de imágenes son árboles de decisiones, redes neuronales y máquinas de vectores de soporte. Sin olvidar que otras son utilizadas, apareciendo novedades más sofisticadas con el devenir del tiempo, incluyendo algunas que por su estrategia para los servicios de seguridad no son públicas.

Asimismo, el uso de la clasificación y aprendizaje automáticos es diversa, buscando, como es evidente, automatizar procesos repetitivos, de inspección y vigilancia compleja, entre otros asuntos.

Finalmente, dejar patente que estos procesos son cada vez más utilizados y su desarrollo en el futuro, además de estratégico, va a significar un avance para cuestiones tales como el Internet de las cosas, como paradigma de la cotidianidad con las que vamos a convivir con ellas.

\section{Referencias}

Al-Batah, M. S., Isa, N. A. M., Zamli, K. Z., Sani, Z. M., y Azizli, K. A. (2009). A novel aggregate classification technique using moment invariants and cascaded multilayered perceptron network. International Journal of Mineral Processing, 92(1-2), 92-102.

Amutha, A. L., y Kavitha, S. (2011). Features based classification of images using weighted feature support vector machines. Int J Comput Appl, 26(10), 23-9.

Augereau, O., Journet, N., Vialard, A., y Domenger, J.-P. (2014). Improving classification of an industrial document image database by combining visual and textual features. En Document Analysis Systems (DAS), 2014 11th IAPR International Workshop on (pp. 314-318). IEEE.

Cintra, D. P., Novack, T., Rego, L. F. G., Costa, G., y Feitosa, R. Q. (2010). PIMAR ProjectMonitoring the atlantic rainforest remnants and the urban growth of the Rio de Janeiro city (Brazil) through remote sensing. The International Archives of the Photogrammetry, Remote Sensing and Spatial Information Sciences, XXXVIII-4/C7.

Correia, R., Duarte, L., Teodoro, A., y Monteiro, A. (2018). Processing Image to Geographical Information Systems (PI2GIS)—A Learning Tool for QGIS. Education Sciences, 8(2), 83. 
Das, A.J. Saikia, N. y Sarma, K.K. (2016). Object classification and tracking in real time: an overview. P. 250-295. En Santhi, V., Acharjya, D.P. y Ezhilarasan, M. (eds.). Emerging technologies in intelligent applications for image and video processing. Hershey, PA: IGI Global.

Dimitrios, A., Rousopoulos, P., Papaodysseus, C., Panagopoulos, M., Loumou, P., y Theodoropoulos, G. (2010). A general methodology for the determination of 2D bodies elastic deformation invariants: Application to the automatic identification of parasites. IEEE transactions on pattern analysis and machine intelligence, 32(5), 799-814.

Ghaffarian, S. (2014). Automatic building detection based on supervised classification using high resolution Google Earth images. The International Archives of Photogrammetry, Remote Sensing and Spatial Information Sciences, 40(3), 101.

HaCohen-Kerner, Y., Sabag, A., Liparas, D., Moumtzidou, A., Vrochidis, S., y Kompatsiaris, I. (2015). Classification Using Various Machine Learning Methods and Combinations of Key-Phrases and Visual Features. En Semanitic Keyword-based Search on Structured Data Sources (pp. 64-75). Springer.

Hao Z., Ge H. y Wang, L. (2018) Visual attention mechanism and support vector machine based automatic image annotation. PLOS ONE, 13(11).

Hemsley, A., y Mukundan, R. (2009). Multifractal Measures for Tissue Image Classification and Retrieval. 11th IEEE International Symposium on Multimedia(ISM), San Diego, California, pp. 618-623.

Hermosilla, T., Ruiz, L. A., Recio, J. A., y Estornell, J. (2011). Evaluation of automatic building detection approaches combining high resolution images and LiDAR data. Remote Sensing, 3(6), 1188-1210.

Hughes, N. (2009). Sea ice type classification from multichannel passive microwave datasets. En Geoscience and Remote Sensing Symposium, 2009 IEEE International, IGARSS 2009 (Vol. 3, pp. III-125). IEEE.

Jabari, S. y Zhang, Y. (2013). Very high resolution satellite image classification using fuzzy rulebased Systems. Algorithms, 6, 762-781.

Jain, S. (2013). A machine learning approach: Svm for image classification in cbir. Internationa Journal of Aplication or Annovation in Engineering \& Management (IJAIEM), 2(4).

Kotsiantis, S.B. (2007). Supervised machine learning: A review of classification techniques. Informatica, 31, 249-268.

Kupidura, P., Osińska-Skotak, K., y Pluto-Kossakowska, J. (2016). Automatic Approach to Vhr Satellite Image Classification. ISPRS-International Archives of the Photogrammetry, Remote Sensing and Spatial Information Sciences, 277-282. 
Li, B., Tan, X., Wang, F., Lian, P., Gao, W., y Li, Y. (2017). Fracture and vug characterization and carbonate rock type automatic classification using X-ray CT images. Journal of Petroleum Science and Engineering, 153, 88-96.

Lin, Y., Li, W. J., Yu, J., y Wu, C. Z. (2018). Ecological Sensitivity Evaluation of Tourist Region Based on Remote Sensing Image-Taking Chaohu Lake Area as a Case Study. International Archives of the Photogrammetry, Remote Sensing \& Spatial Information Sciences, 42(3).

Ma, L, Zeheng, G., Eitel, J. y Moskal, L.M. (2016). Improved Salient Feature-Based Approach for Automatically Separating Photosynthetic and Nonphotosynthetic Components Within Terrestrial Lidar Point Cloud Data of Forest Canopies. IEEE Transactions on geoscience and remote sensing, 54(2), 679-696.

Minetto, R. Thome, N., Cord, M., Leite, N.J. y Stolfi, J. (2014). SnooperText: A text detection system for automatic indexing of urban scenes. Computer Vision and Image Understanding, 122, 92-104.

Morioka, C., Meng, F., Taira, R., Sayre, J., Zimmerman, P., Ishimitsu, D. El-Saden, S. (2016). Automatic classification of ultrasound screening examinations of the abdominal aorta. Journal of digital imaging, 29(6), 742-748.

Murphy, J. M., y Maggioni, M. (2018). Unsupervised Clustering and Active Learning of Hyperspectral Images With Nonlinear Diffusion. IEEE Transactions on Geoscience and Remote Sensing.

Ping Tian, D. (2013). A review on image feature extraction and representation techniques. International Journal of Multimedia and Ubiquitous Engineering, 8(4), 385-396.

Raj, K. J., y SivaSathya, S. (2016). A Survey of Various Algorithms Used on Multispectral Satellite Image Classification of Alwar Image Dataset. Indian Journal of Science and Technology, 9(45).

Rezaeian, M. (2012). Automatic classification of collapsed buildings using stereo aerial images. International Journal of Computer Applications, 46(21), 35-42.

Senthilnath, J., Kulkarni, S., Benediktsson, J. A., y Yang, X.-S. (2016). A novel approach for multispectral satellite image classification based on the bat algorithm. IEEE Geoscience and Remote Sensing Letters, 13(4), 599-603.

Shen, J. (2009). Stochastic modeling western paintings for effective classification. Pattern Recognition. 42(2), 293-301.

Tian, Dong Ping (2013). A Review on image feature extraction and representation techniques. International Journal of Multimedia and Ubiquitous Engineering, 8(4), 385-396.

Velazco Paredes, Y.E. (2014). Recuperación de imágenes por contenido basado en regiones con retroalimentación por relevancia. Universidad Nacional de San Agustín de Arequipa. Tesis doctoral. Disponible en : http://repositorio.unsa.edu.pe/handle/UNSA/6129 [Consultado: 05-11-2018]. 
Yang, M. D., Huang, K. S., Kuo, Y. H., Tsai, H. P., \& Lin, L. M. (2017). Spatial and spectral hybrid image classification for rice lodging assessment through UAV imagery. Remote Sensing, 9(6), 583.

You, Y., Wang, S., Ma, Y., Chen, G., Wang, B., Shen, M., y Liu, W. (2018). Building detection from VHR remote sensing imagery based on the morphological building index. Remote Sensing, 10(8), 1287. doi: 10.3390/rs 10081287

Zhang, F. y Zhang, X. (2011). Classification and quality evaluation of tobacco leaves based on image processing and fuzzy comprehensive evaluation. Sensors, 11, 2369-2384.

Zhang, W., Hu, B., Jing, L., Woods, M. E., y Courville, P. (2008). Automatic forest species classification using combined LIDAR data and optical imagery. En Geoscience and Remote Sensing Symposium, 2008. IGARSS 2008. IEEE International (Vol. 3, pp. III-134). IEEE. 